\title{
Negative Optical Torque
}

SUBJECT AREAS:

OPTICAL MANIPULATION

AND TWEEZERS

COMPUTATIONAL

NANOTECHNOLOGY

Received

8 May 2014

Accepted

6 August 2014

Published

17 September 2014

Correspondence and requests for materials should be addressed to

J.N. (jacktfng@hkbu. edu.hk) or C.T.C. (phchan@ust.hk)

* These authors contributed equally to this work.
Jun Chen ${ }^{1,2,3 *}$, Jack Ng${ }^{4 *}$, Kun Ding ${ }^{1,5}$, Kin Hung Fung ${ }^{6}$, Zhifang $\operatorname{Lin}^{3} \&$ C. T. Chan ${ }^{1,5}$

'Department of Physics, The Hong Kong University of Science and Technology, ${ }^{2}$ Institute of Theoretical Physics and Department of Physics, Shanxi University, ${ }^{3}$ State Key Laboratory of Surface Physics, Key Laboratory of Micro and Nano Photonic Structures (MOE), and Department of physics, Fudan University, ${ }^{4}$ Department of Physics and Institute of Computational and Theoretical Studies, Hong Kong Baptist University, ${ }^{5}$ Institute for Advanced Study, The Hong Kong University of Science and Technology, ${ }^{6}$ Department of Applied Physics, The Hong Kong Polytechnic University.

Light carries angular momentum, and as such it can exert torques on material objects. Applications of these opto-mechanical effects were limited initially due to their smallness in magnitude, but later becomes powerful and versatile after the invention of laser. Novel and practical approaches for harvesting light for particle rotation have since been demonstrated, where the structure is always subjected to a positive optical torque along a certain axis if the incident angular momentum has a positive projection on the same axis. We report here an interesting phenomenon of "negative optical torque", meaning that incoming photons carrying angular momentum rotate an object in the opposite sense. Surprisingly this can be realized quite straightforwardly in simple planar structures. Field retardation is a necessary condition and discrete rotational symmetry of material object plays an important role. The optimal conditions are explored and explained.

he negative optical torque (NOT) phenomenon manifestly conserves angular momentum ${ }^{1-4}$. Let us consider an object illuminated by an electromagnetic wave carrying a $z$-component angular momentum of $L_{z}^{\text {inc }}$. After being scattered by the object, the total $z$-component angular momentum of the light is $L_{z}^{s c a}$. If the consequence of scattering is such that $L_{z}^{s c a}>L_{z}^{i n c}$, then angular momentum conservation requires that the difference $L_{z}^{s c a}-L_{z}^{i n c}$ must be balanced by a NOT acting on the object. Consequently, negative optical torque is consistent with total angular momentum conservation. The question is then whether such phenomenon, which does not violate conservation laws, can be realized in practice. If so, is NOT easily observable? We will show that NOT is in fact ubiquitous, as it can be realized in many transparent or weakly absorptive structures, in discrete rotationally symmetric or even irregular structures, and also in planar or even 3D structures. We shall show that field retardation is necessary for achieving NOT and discrete rotational symmetry enhances NOT. The realization of NOT adds new possibility to the existing novel ${ }^{5-12}$ and practical ${ }^{13-21}$ technique of optical rotation. It may open up new applications in areas where optical micromanipulation play a role $e^{22,23}$. We note that although negative optical torque here is indeed the angular counterpart of optical pulling force ${ }^{24-27}$, it is definitely not a straight forward corollary. For example, symmetry is not a key factor in achieving optical pulling force but it proved to be of considerable importance in realizing NOT. Also, NOT is ubiquitous while optical pulling force can only be observed with a carefully chosen combination of beam profile and particle properties.

\section{Results}

Explicit numerical demonstration of negative optical torque. Explicit examples of structures where NOT can be realized are shown in Fig. 1. For the ease and accuracy of computation and interpretation, we consider planar structures composing of dielectric microspheres located on the $x y$-plane. The axis for which the torque is to be calculated is parallel to the $z$-axis and goes through the center of mass marked in Fig. 1, where some of the structures possess discrete rotational symmetry but some do not. We stress that our conclusions are valid not only for discrete system comprising of spheres, but also to a continuous piece of material.

The time-averaged optical torque $\Gamma$ acting on the concerned structure is computed by (see Methods)

$$
\boldsymbol{\Gamma}=\iint_{\sigma}(\mathbf{r} \times\langle\overleftrightarrow{\mathbf{T}}\rangle) \cdot \hat{\mathbf{n}} d S .
$$

where the time averaged Maxwell stress tensor is given by ${ }^{28}$

$$
\langle\overleftrightarrow{\mathbf{T}}\rangle=\frac{\varepsilon_{0}}{2}\left[\mathbf{E E}^{*}+c^{2} \mathbf{B} \mathbf{B}^{*}-\frac{1}{2}\left(|\mathbf{E}|^{2}+c^{2}|\mathbf{B}|^{2}\right) \mathbf{I}\right],
$$




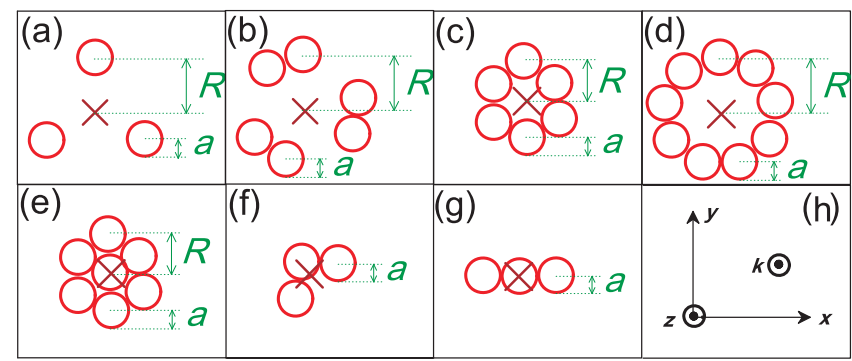

Figure 1 Examples of planar structures that can realize negative optical torque. (a)-(g) structures composed of an array of identical dielectric spheres. $R(a)$ is the structure (sphere) radius. The crosses mark the centers of mass, which coincides with the axis of rotation. (h) shows the coordinate system. The incident plane wave has a k-vector $(k)$ along the $\mathrm{z}$-axis.

the origin of the coordinate system is chosen to be the center of mass marked in Fig. 1, $\sigma$ is any surface that encloses the entire structure, and $\mathbf{r}$ is the position vector originating from the center of mass. Irrespective of the structural orientation and morphology, the $z$ component optical torque calculated by Eq. (1) can be written as

$$
\Gamma_{z}=\sum_{m_{i}} \frac{m_{i} \hbar}{\hbar \omega} W_{e x t}^{\left(m_{i}\right)}-\sum_{m} \frac{m \hbar}{\hbar \omega} W_{s c a}^{(m)},
$$

where $\omega$ is the angular frequency of incident wave, and $m_{i}$ and $m$ characterize, respectively, the azimuthal channels for the incident and scattered waves. $W_{\text {ext }}^{\left(m_{i}\right)}$ is the energy extinction rate for incident wave from channel $m_{i}$, and $W_{s c a}^{(m)}$ is the rate at which energy is scattered into the outgoing channel $m$. The derivation and the explicit expressions of $W_{e x t}^{\left(m_{i}\right)}$ and $W_{s c a}^{(m)}$ are given in the Methods section. As long as the scattering problem can be treated accurately, Eq. (3) is exact within the framework of classical electrodynamics. In the numerical results shown below, we will consider objects composing of an array of spheres as our prototypical system because the scattering problem can be solved numerically using the generalized Mie theory for multi-sphere to a very high precision. This removes any doubt that the NOT effects observed are numerical artifacts. And unless stated otherwise, the incident wave is a left circularly polarized $^{28}$ plane wave propagating along the $z$-axis, with a positive angular momentum and an intensity of $1 \mathrm{~mW} / \mu \mathrm{m}^{2}$.

Figure 2 shows the calculated optical torques versus $k a$ for three morphologically different planar structures with different types of incident waves, where $k$ is the wave number and $a$ is the radius of the comprising spheres. The results clearly show that NOT can be realized in all three structures, which include planar triangular or hexagonal structures consisting of silicon or polystyrene microspheres, illuminated by a circularly polarized plane wave, Gaussian beam, or Laguerre Gaussian beam. The optical torques can take negative values at some intervals of $k a$. The three structures in Fig. 2 are not of special design. Other planar structures can serve the same purpose. Figure 2 illustrates that NOT can be quite universal: it can be observed in different structures (planar or non-planar) with different forms of incident wave, including plane wave, Gaussian beam, and Laguerre Gaussian beam. For the Laguerre Gaussian beam, the smaller structure (yellow line) do not experience a NOT as the beam cannot probe the structural details. While for the larger structure (blue line), NOT is still seen.

Analytical demonstration of negative optical torque and the role of retardation. In addition to numerical demonstration, formal analytical understanding of NOT can be developed by considering the following prototypical system: a pair of non-absorbing dipolar spheres located on the $x y$-plane, illuminated by a $z$-propagating plane wave with arbitrary polarization. Following Ref. 24 and

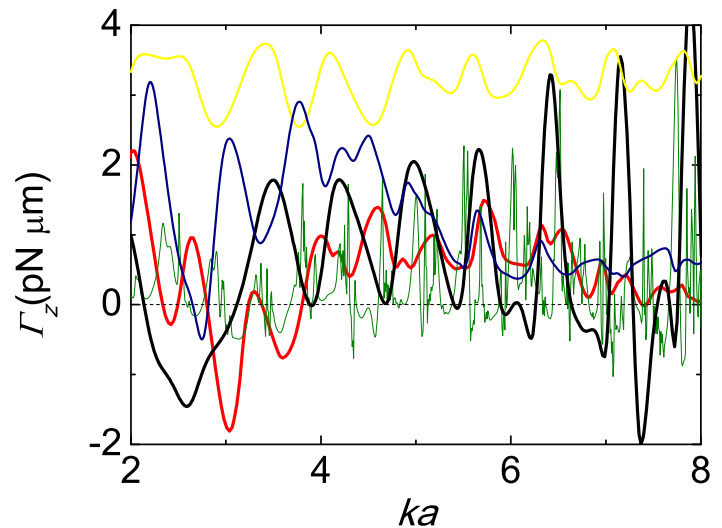

Figure $2 \mid$ Optical torque for different structures under different incident waves carrying positive angular momentum. Red: Optical torque versus varying $k$ for Fig. $1(\mathrm{e})\left(\varepsilon_{r}=2.46, a=0.49 \mu \mathrm{m}, R=1.0 \mu \mathrm{m}\right)$ illuminated by a left circularly polarized plane wave of intensity $1 \mathrm{~mW} / \mu \mathrm{m}^{2}$. Green: Optical torque versus varying $a$ for Fig. 1 (a) composed of silicon spheres $\left(\varepsilon_{r}\right.$ $=12.6096, R=1.16 a, \lambda=1064 \mathrm{~nm}$ ) in water illuminated by a left circularly polarized plane wave of intensity $=1 \mathrm{~mW} / \mu \mathrm{m}^{2}$. Black: Optical torque versus varying $k$ for Fig. 1 (a) $\left(\varepsilon_{r}=2.4649, a=0.5 \mu m, R=\right.$ $0.58 \mu \mathrm{m})$ illuminated by a left circularly polarized Gaussian beam (numerical aperture $=0.9$ and total power $=0.05 \mathrm{~W}$ ). Yellow: Optical torque versus $k$ for Fig. 1(a) $\left(\varepsilon_{r}=2.4649, a=0.5 \mu \mathrm{m}, R=0.58 \mu \mathrm{m}\right)$ illuminated by a linearly polarized $10 \mathrm{~mW}$ Laguerre-Gaussian beam with topological charge of 1 and a N.A. of 0.9. Blue: Optical torque versus $k$ for Fig. 1(e) $\left(\varepsilon_{r}=2.4649, a=0.49 \mu \mathrm{m}, R=1.0 \mu \mathrm{m}\right)$ illuminated by a linearly polarized $10 \mathrm{~mW}$ Laguerre-Gaussian beam with topological charge of 1 and a N.A. of 0.9. Negative torque $\left(\Gamma_{z}<0\right)$ means that the light beam induces the object to rotate in the opposite sense as the angular momentum of the incident photon.

retaining only the electric dipole term, one can derive the formula for optical torque:

$$
\Gamma_{z}=\frac{|\alpha|^{2}}{2} \operatorname{Re}\left(\mu_{\|}-\mu_{\perp}\right)\left[\begin{array}{c}
\operatorname{Re}\left(E_{\|} E_{\perp}^{*}\right) \operatorname{Re}\left(1+\alpha \mu_{\perp}+\alpha \mu_{\|}\right) \\
+\left(\left|E_{+}\right|^{2}-\left|E_{-}\right|^{2}\right) \operatorname{Im}\left(\alpha \mu_{\|}-\alpha \mu_{\perp}\right)
\end{array}\right],
$$

where

$$
\begin{aligned}
& \mu_{\|}=\frac{2 e^{i k R}}{4 \pi \varepsilon_{0} R^{3}}(1-i k R), \\
& \mu_{\perp}=\frac{e^{i k R}}{4 \pi \varepsilon_{0} R^{3}}\left(k^{2} R^{2}+i k R-1\right)
\end{aligned}
$$

are the eigenvalues of the dyadic Green's function ${ }^{29}, R$ is the separation between the dipoles, and $\alpha$ is the dipolar polarizability. $E_{\|}$and $E_{\perp}$ are the projection of the electric field parallel to or perpendicular to the particle axis (i.e. the amplitudes of linear polarization), $E_{+}$and $E_{-}$are the amplitudes of left and right circularly polarized components. The effect of field retardation manifests itself as a factor of $e^{i k R}$ in the expression of $\mu_{\|}$and $\mu_{\perp}$, leading to oscillatory behavior of toque versus $k R$. We shall see that it is this oscillation due to retardation that opens up the possibility of NOT.

The first term in Eq. (4) is proportional to $\operatorname{Re}\left(E_{\|} E_{\perp}^{*}\right)$. It is an aligning force, which vanishes for circular polarization. For linearly polarization, it vanishes only when either $E_{\|}=0$ or $E_{\perp}=0$, corresponding to the case of polarization being perpendicular or parallel to the particle axis, respectively. Accordingly, depending on $k R$, this force tries to align the particle chain to a direction that is either perpendicular or parallel to the polarization.

The second term in Eq. (4), which is proportional to $\left(\left|E_{+}\right|^{2}-\right.$ $\left.\left|E_{-}\right|^{2}\right)$, also has a simple interpretation: the sign of the corresponding 
torque is determined by the competition between the amplitudes of the left and right circular polarization. Consequently, it is a rotating force that will keep the object turning. Depending on $k R$, a left circularly polarized light can induce a positive or negative torque. Consequently, this term is responsible for NOT. Moreover, the term vanishes identically for linear polarization.

The two terms in Eq. (4) are competing with each other. When the incident wave is predominantly linearly polarized, the first aligning term will be dominating and the particle chain will be aligned. On the other hand, for circular polarization, the second turning term will dominate and the particle chain will be set into rotation. Equation (4) contains many salient features of light induced torque. For circular polarization, it becomes

$$
\Gamma_{z}=\frac{\left|\alpha^{2}\right|}{2}\left(\left|E_{+}\right|^{2}-\left|E_{-}\right|^{2}\right) f_{1}[\operatorname{Im} \alpha+|\alpha| \sin (2 k R+\delta)],
$$

where $\Gamma_{x}=\Gamma_{y}=0$ by symmetry,

$$
f_{1}=\frac{k^{4} R^{4}+3 k^{2} R^{2}+9}{32 \pi^{2} R^{6} \varepsilon_{0}^{2}}
$$

and

$$
\delta=\tan ^{-1}\left[\frac{\left(6 k^{3} R^{3}-18 k R\right) \operatorname{Re}\{\alpha\}+\left(9-15 k^{2} R^{2}+k^{4} R^{4}\right) \operatorname{Im}\{\alpha\}}{\left(9-15 k^{2} R^{2}+k^{4} R^{4}\right) \operatorname{Re}\{\alpha\}-\left(6 k^{3} R^{3}-18 k R\right) \operatorname{Im}\{\alpha\}}\right] .
$$

The first term inside the square bracket of Eq. (6) corresponds to a positive bias, whereas the second term carrying $\sin (2 k R+\delta)$ oscillates between positive and negative values due to the spatial field retardation associated with a finite value of $k R$. Since $|\alpha|>\operatorname{Im} \alpha$, the amplitude of oscillation is larger than that of the bias, accordingly, the optical torque can sometimes be negative due to spatial retardation induced oscillation. This simple exercise not only demonstrates analytically the possible existence of NOT, the results also show that field retardation is essential to realize NOT.

For very small separations between the dipoles and a left circularly polarized incident wave, Eq. (6) asymptotically approaches

$$
\Gamma_{z}=\left|\alpha^{2}\right| E_{0}^{2} \frac{9 \operatorname{Im} \alpha}{32 \pi^{2} \varepsilon_{0}^{2} R^{6}} .
$$

Since $\operatorname{Im} \alpha>0$ by optical theorem ${ }^{30}, \Gamma_{z}>0$. This result is not surprising. In the long wavelength limit $(k R \ll 1)$, the wave cannot probe the details of the structure. Therefore the sign of the torque must be the same as that of the incident wave. In fact, this conclusion holds true for any small structures, as we shall see in the following sessions.

Physical origin of the negative optical torque. We now analyze the physical origin of NOT for more general structures. Equation (3) allows for a heuristic physical interpretation: $W_{e x t}^{\left(m_{i}\right)} / \hbar \omega$ is the extinction rate for incoming photons from channel $m_{\mathrm{i}}$, and each of these photons carries $m_{i} \hbar$ of angular momentum, therefore the rate at which the object acquires angular momentum from all azimuthal channels of the incident beam is given by the first term in Eq. (3). Similarly $W_{s c a}^{(m)} / \hbar \omega$ is the rate of photons being scattered into channel $m$, and each of these photons gives a recoil angular momentum of $-m \hbar$, therefore the rate at which the object obtains angular momentum due to recoil is given by the second term in Eq. (3). Equation (3) also shows that the transfer of angular momentum can be interpreted as occurring in two steps. First, the photons are intercepted by the scatterer. This induces an extinction torque given by the first term in Eq. (3). For an incident wave consisting of solely positive $m_{i}$ components, this extinction torque is positive definite. So if NOT exists at all, it must come from the recoil torque in Eq. (3). Depending on the material and the structural properties of the object, some of the intercepted photons will be absorbed, while some will be scattered. The scattered photons exert a recoil toque on the object, as given by the second term in Eq. (3), where $m<0(m>0)$ represent a positive (negative) contribution. If the negative contribution dominates, we will have a NOT.

The role of symmetry. Symmetry is the explanation to why the negative optical torque effect can actually be quite universal. Light cannot exert an optical torque on a transparent object possessing continuous rotational symmetry, since photons scattered by such an object preserve their angular momentum. From this point of view, the phenomenon of optical torque is a consequence of broken rotational symmetry. NOT is therefore inevitably tied to the symmetry of the structure and incident wave.

To see this, it suffices to consider objects with discrete rotational symmetry of degree $m_{s}$ and for an incident wave with a single azimuthal channel $m_{i}>0$. In wave scattering theory, based on irreducible tensor approach, wave can be decomposed into modes characterized by two mode indices: total angular momentum index $l$, termed also angular momentum channel index, and $z$-component of angular momentum index $m$, termed also azimuthal channel index in this paper. We will see that it is discrete rotational symmetry that allows NOT to be observed ubiquitously.

The angular momentum selection rule for photons after being scattered by such object is

$$
m=m_{i}+n \times m_{s}
$$

where $m$ is an allowed channel for the scattered wave and $n=0, \pm 1$, \pm 2 and so on ${ }^{31,32}$ (see part 1 of Methods for a proof). Equation (10) is the angular counterpart of the grating effect for a periodic surface illuminated by a plane wave. Although Eq. (10) is analogous to linear grating (periodic in space), the rules are correct for all structures in the rotational case because any structure is invariant under a rotation of $2 \pi$. With Eq. (10), the $z$-component optical torque of Eq. (3) can be re-written as

$$
\Gamma_{z}=\frac{m_{i} \hbar}{\hbar \omega} W_{e x t}^{\left(m_{i}\right)}-\sum_{n}\left(m_{i}+n \times m_{s}\right) \frac{\hbar}{\hbar \omega} W_{s c a}^{\left(m_{i}+n \times m_{s}\right)} .
$$

In principle, the positive and negative $n$ channels contribute to NOT and positive optical torque (POT), respectively, while the $n=0$ channel, representing no change in the angular momentum of the photons, produces no torque. However, the contribution for channels with $|m| \gg k R$ can be rather weak. When $|m| \gg k R$, the centrifugal effect forces the amplitude of the mode $m$ to decrease rapidly for region where $R<|m| / k^{33}$. There is very little overlap between the mode $m$ and the object, forbidding the object to scatter wave into these channels. Consequently, these channels contribute negligibly to optical torque.

If $m_{\mathrm{s}}$ is very large, the condition of $|m| \gg k R$ will be met for all channel except the $n=0$ channel, even for the low order modes with $n= \pm 1$. In fact, continuous rotational symmetry is an idealization rather than reality. An object with very fine sub-wavelength details (such as a small object with a large $m_{\mathrm{s}}$ ) that is beyond the diffraction limited resolving power of the incoming wave can be thought to have continuous rotational symmetry as far as light scattering is concerned. In this case, the contributions from all channels become vanishingly small except the $n=0$ channel. Therefore the wave exerts vanishingly small torque on such object with high discrete rotational symmetry and relatively small size, just like a structure with continuous rotational symmetry. The incident wave $m_{i}$ can only be scattered into the azimuthal channel characterized by the same $m_{i}$. Equation (3) thus reduces to $\Gamma_{z}=\sum_{m_{i}} m_{i} \hbar W_{a b s}^{\left(m_{i}\right)} / \hbar \omega$, where $W_{\mathrm{abs}}^{\left(m_{i}\right)}=W_{\mathrm{ext}}^{\left(m_{i}\right)}-W_{\mathrm{sca}}^{\left(m_{i}\right)}$ is proportional to the energy absorption rate in channel $m_{i}$. Since both $W_{a b s}^{\left(m_{i}\right)}$ and $m_{i}$ are positive definite, it follows that $\Gamma_{z} \geq 0$. 
In the other extreme situation of $m_{s} \ll m_{i}$, according to Eq. (3), the torque is positive definite as

$$
\begin{aligned}
\Gamma_{z} & \approx m_{i} / \omega\left(W_{e x t}^{\left(m_{i}\right)}-\sum_{n} W_{s c a}^{\left(m_{i}+n \times m_{s}\right)}\right) \\
& =m_{i} \frac{\left(W_{e x t}-W_{s c a}\right)}{\omega}=\frac{m_{i} W_{a b s}}{\omega} \geq 0,
\end{aligned}
$$

where $W_{a b s}=\left(W_{\text {ext }}-W_{\text {sca }}\right)$ is the rates at which energy is absorbed.

If NOT can exist, it must be for some moderate value of $m_{s}<k R$ so that the corresponding partial waves can reach the scatterer to transfer angular momentum. This is in fact a manifestation of the optical diffraction limit. If the small structure has some very fine subwavelength details, such as patterns with high degree of rotational symmetry located in a small region, the incident wave will not be able to probe them. Thus the information on the symmetry will be lost, eliminating the possibility of observing NOT. In general, NOT can be easily observed if $m_{s}<l_{\max }$, where $l_{\max }=k R+4.05(k R)^{1 / 3}+2$ is an empirical formula for the angular momentum truncation order of the scatterer ${ }^{31,32}$.

Next, we argue that a larger $m_{s}$ favors NOT as long as $|m|$ is not significantly larger than $k R$. In Fig. 3, the optical torque acting on objects composing of the same type of spheres but with different values of $m_{s}\left(m_{s}=1,2,3\right)$ are shown. By comparing $m_{s}=1$ to $m_{s}$ $=3$, one sees that NOT is more easily observed for a larger value of $m_{s}$. These can be explained as follows. We note that according to our simulations, and also Eq. (6), the total torque consists of a positive bias part and an oscillatory part. It is the oscillatory part that gives NOT. For the object with $m_{s}=1$ (red line), the torque acting on all three spheres are different. Therefore the negative torque acting on one sphere may be canceled by the positive torque acting on another. This weakens the oscillatory part of the total torque and reduces the possibility of NOT. For the object with $m_{s}=3$ (blue line), the torque acting on each sphere is the same by symmetry, so the torques reinforce each other rather than cancel each other. This preserves the strength of the oscillatory part of the total torque and allows NOT to be observed more easily. More discussion about the role of symmetry can be found in the Supplementary Information.

\section{Discussion}

For the more complex systems we have considered (arrays of spheres as shown in Fig. 1), we further argue here that spatial retardation is also essential for NOT, as in the simple example we discussed in the previous section. We show in Fig. 4 the optical torque due to indi-

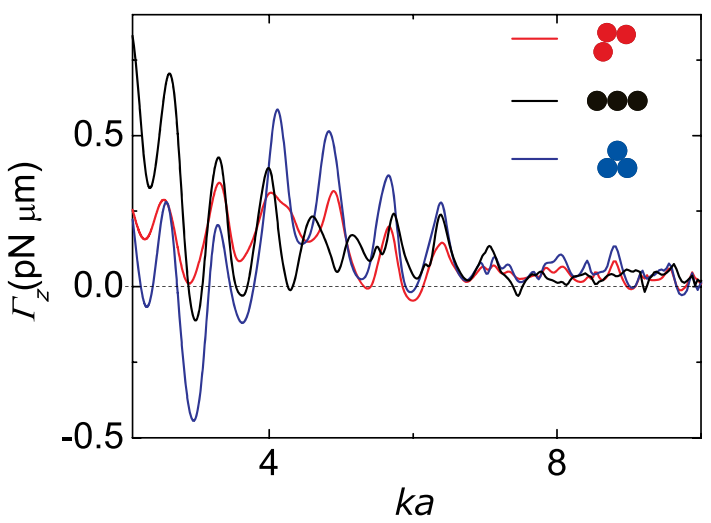

Figure 3 Optical torque versus $k$ a for objects with different discrete rotational symmetry. The objects are composed of identical dielectric spheres $\left(\varepsilon_{r}=2.4649\right)$. The red, black, and blue lines correspond to $m_{s}=1$, 2 , and 3 , respectively. The degree of discrete rotational symmetry is seen to significantly enhance negative optical torque (i.e. higher $m_{s}$ favors negative torque). vidual azimuthal channel for structures with three-fold $\left(m_{s}=3\right)$ or six-fold $\left(m_{s}=6\right)$ rotational symmetry. According to Eq. (10), for three-fold symmetric structures showed in Fig. 4(a)-(b), light is scattered into azimuthal channels characterized by $m=3 n+1$, where $n$ $=0, \pm 1, \pm 2, \ldots$ etc. The $m=1$ channel [i.e. $n=0$ in Eq. (10)] does not contribute to torque for lossless spheres. Light scattered into azimuthal channels $m=-2,-5,-8$ and so on will contribute to positive optical torque, whereas the channels characterized by $m=4$, 7,10 and so on will induce NOT. As multiple scattering is not strong in dielectrics, the recoil torque is dominated by $n= \pm 1$, corresponding to $m=m_{i} \pm m_{s}=-2$ or 4 . In Fig. 4 the oscillatory behavior of the torques due to individual partial waves versus wavelength and structural size is the signature of retardation, while the peaks for $n= \pm 1$ channels correspond to Mie type resonance. It is precisely due to this retardation induced oscillation that the total torque takes positive or negative value. The situation for the six-fold symmetric structures showed in Fig. 4(c)-(d) is similar.

Finally, we argue that small object does not favor NOT. Consider, for example, a small three-fold $\left(m_{s}=3\right)$ rotationally symmetric lossless structure illuminated by a left circularly polarized plane wave $\left(m_{i}=1\right)$. If the structure is small such that wave components with higher angular momentum cannot reach the scatterer and the truncation angular momentum index $l_{\max }=1$, then the torque is approximately equal to zero since only $n=0$ term appears in Eq. (11). As the size of the structure increases such that we have to take $l_{\max }=2$, the second term in the series (corresponding to $n=-1$ ) is now significant, which is given by (see Methods for the definition of partial torque $\tau_{m}$ that is due to each individual outgoing azimuthal channel $m$ )

$$
\tau_{-2}=m_{s} W_{-2,2} / \omega .
$$

Equation (13) is positive definite and therefore corresponds to POT. One then expects that a small scatterer cannot experience a NOT. If we consider larger particle, NOT will become possible. For example, the third term in the series (corresponding to $n=1$ ) is given by

$$
\tau_{4}=-m_{s} W_{4,4} / \omega
$$

which, when larger in magnitude than $\tau_{-2}$ in Eq. (13), results in negative total torque.

In summary, we showed that negative optical torque is ubiquitous in simple configurations and can be induced by a broad class of incident waves. We note that while negative optical torque can be viewed as the angular counterpart of the optical pulling force, the conditions under which the "negative response" can be realized are very different. The optical pulling force is achievable only for special kinds of optical beams acting on some specific particles ${ }^{24-27}$ whereas NOT can be realized rather easily. NOT can occur if the recoil torque happens to be negative and larger in magnitude than the extinction torque, which is made possible by retardation effects and enhanced by discrete rotational symmetry. We remark that our theory is valid for both spin and orbital angular momentum (i.e. arbitrary $m_{i}$ ) of light.

\section{Methods}

Derivation of the azimuthal mode selection rule in electromagnetic scattering. We shall prove that when an incident wave characterized by a single azimuthal number $m_{i}{ }^{30,32}$ impinges on a structure with $m_{s}$-fold rotational symmetry, it will be scattered into azimuthal channels given by the azimuthal mode selection rule in Eq. (10).

Let us start with the total electric field for a general scattering problem, which is given by the Lippmann-Schwinger integral equation ${ }^{34,35}$

$$
\mathbf{E}(\mathbf{r})=\mathbf{E}_{\mathrm{inc}}(\mathbf{r})+\int \overleftrightarrow{\mathbf{G}}\left(\mathbf{r}, \mathbf{r}^{\prime}\right) \cdot\left[\varepsilon_{r}\left(\mathbf{r}^{\prime}\right)-1\right] k^{2} \mathbf{E}\left(\mathbf{r}^{\prime}\right) \mathrm{d} \mathbf{r}^{\prime},
$$

where $\mathrm{E}_{\text {inc }}(\mathbf{r})$ denotes the incident electric field, $\varepsilon_{r}\left(\mathbf{r}^{\prime}\right)$ is the position dependent relative dielectric constant, and $k=\omega / c$ is the wave number in the background. The second term on the right hand side of Eq. (15) gives the scattered field: 

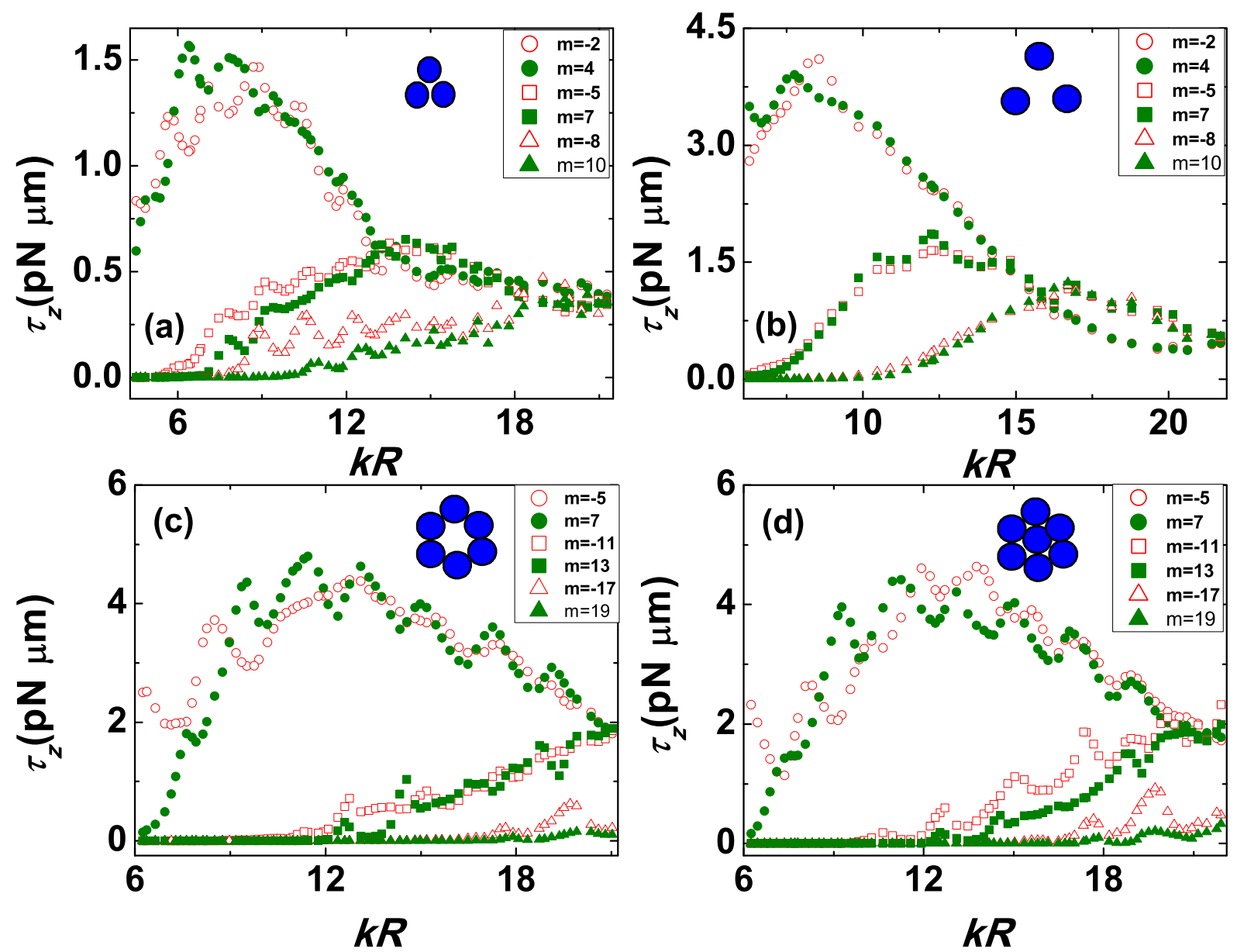

Figure $4 \mid$ The contribution (in absolute value) to the torque by individual partial wave azimuthal channels versus $k R$. (a) -(b) three-fold and (c)-(d) six-fold rotationally symmetric dielectric structures $\left(\varepsilon_{r}=2.4649, a=0.49 \mu \mathrm{m}\right)$. The incident field is a left circularly polarized plane wave (intensity is $\left.1 \mathrm{~mW} / \mu \mathrm{m}^{2}\right) . \mathrm{R}=0.58 \mu \mathrm{m}$ for (a) and $\mathrm{R}=1 \mu \mathrm{m}$ for (b) $-(\mathrm{d})$. The oscillations are due to spatial retardation.

$$
\mathbf{E}_{\mathrm{sca}}=\int \overleftrightarrow{\mathbf{G}}\left(\mathbf{r}, \mathbf{r}^{\prime}\right) \cdot\left[\varepsilon_{r}\left(\mathbf{r}^{\prime}\right)-1\right] k^{2} \mathbf{E}\left(\mathbf{r}^{\prime}\right) \mathrm{d} \mathbf{r}^{\prime}
$$

where $\overleftrightarrow{\mathbf{G}}\left(\mathbf{r}, \mathbf{r}^{\prime}\right)$ is the free-space dyadic Green's function ${ }^{29}$, which can be written as

$$
\overleftrightarrow{\mathbf{G}}\left(\mathbf{r}, \mathbf{r}^{\prime}\right)=\sum_{\alpha, l, m} a_{\alpha m n} \mathbf{O}_{\alpha m l}(r, \theta, \phi) \mathbf{O}_{\alpha(-m) l}\left(r^{\prime}, \theta^{\prime}, \phi^{\prime}\right),
$$

where $\mathbf{O}_{a m n}$ represents vector spherical wave functions $\mathbf{M}_{m n}, \mathbf{N}_{m n}$ and $\mathbf{L}_{m n}{ }^{29,36}$ for $\alpha=$ 1,2 , and 3 , respectively. The $\phi$-dependent part of $\mathbf{O}_{\alpha m l}(r, \theta, \phi)$ is of the form $e^{i m \phi}$ and similarly $\mathbf{O}_{\alpha(-m) l}\left(r^{\prime}, \theta^{\prime}, \phi^{\prime}\right)$ depends on $\phi^{\prime}$ through $e^{-i m \phi^{\prime}}$. As a result, each term characterized by $m$ in the dyadic Green function $\overleftrightarrow{\mathbf{G}}\left(\mathbf{r}, \mathbf{r}^{\prime}\right)$ given by Eq. (17) shows $e^{i m\left(\phi-\phi^{\prime}\right)}$ azimuthal dependence.

Inserting Eq. (15) into Eq. (16), we arrive at

$$
\begin{aligned}
\mathbf{E}_{\text {sca }}= & \int \overleftrightarrow{\mathbf{G}}\left(\mathbf{r}, \mathbf{r}^{\prime}\right) \cdot\left[\varepsilon_{r}\left(\mathbf{r}^{\prime}\right)-1\right] k^{2} \mathbf{E}_{\text {inc }}\left(\mathbf{r}^{\prime}\right) \mathrm{d} \mathbf{r}^{\prime} \\
& +\int \overleftrightarrow{\vec{G}}\left(\mathbf{r}, \mathbf{r}^{\prime}\right) \cdot \overleftrightarrow{\mathbf{G}}\left(\mathbf{r}^{\prime}, \mathbf{r}^{\prime \prime}\right) \cdot\left[\varepsilon_{r}\left(\mathbf{r}^{\prime}\right)-1\right]\left[\varepsilon_{r}\left(\mathbf{r}^{\prime \prime}\right)-1\right] k^{4} \mathbf{E}_{\text {inc }}\left(\mathbf{r}^{\prime \prime}\right) \mathrm{d} \mathbf{r}^{\prime \prime} \mathrm{d} \mathbf{r}^{\prime} \\
& +\cdots,
\end{aligned}
$$

where $\cdots$ denotes higher order scattering terms. Note that the azimuthal dependence of the incident wave $\mathbf{E}_{\text {inc }}\left(\mathbf{r}^{\prime}\right)$ on $\phi^{\prime}$ is given by $e^{i m_{i} \phi^{\prime}}$, while for a structure with $m_{s}$-fold symmetry, the dielectric constant may be expanded in a Fourier series

$$
\varepsilon_{r}\left(\mathbf{r}^{\prime}\right)-1=\sum_{n=-\infty}^{\infty} A_{n} e^{i n m_{s} \phi^{\prime}} .
$$

Therefore, for the first term on the right hand side of Eq. (18), the integration over azimuthal coordinate $\phi^{\prime}$ reads

$$
\sum_{\alpha, l, m} \cdots \int_{0}^{2 \pi} e^{-i m \phi^{\prime}}\left(\sum_{n} A_{n} e^{i n m_{s} \phi^{\prime}}\right) e^{i m_{i} \phi^{\prime}} d \phi^{\prime}
$$

which requires $m=m_{i}+n m_{s}$ in order to have a non-zero contribution to $\mathbf{E}_{s c a}$. Similarly, for the second term on the right hand side of Eq. (18), the integration over $\phi^{\prime}$ and $\phi^{\prime \prime}$ can be written as

$$
\sum_{\alpha, l, m} \sum_{\alpha^{\prime}, l^{\prime}, m^{\prime}} \cdots \int_{0}^{2 \pi} \int_{0}^{2 \pi} e^{-i m \phi^{\prime}} e^{i m^{\prime}\left(\phi^{\prime}-\phi^{\prime \prime}\right)}\left(\sum_{n, n^{\prime}} A_{n} e^{i n m_{s} \phi^{\prime}} A_{n^{\prime}} e^{i n^{\prime} m_{s} \phi^{\prime \prime}}\right) e^{i m_{i} \phi^{\prime \prime}} d \phi^{\prime} \mathrm{d} \phi^{\prime \prime} .
$$

It then follows straightforwardly that the non-vanishing terms should satisfy $m^{\prime}=$ $m_{i}+n^{\prime} m_{s}$ and $m=m^{\prime}+n m_{s}$, implying that only azimuthal modes with $m=(n+$ $\left.n^{\prime}\right) m_{s}+m_{i}$ survive in the scattered field.

The procedure applies straightforwardly to higher order scattering terms in Eq. (18), leading to the conclusion that the scattered field contains only azimuthal modes with $m$ satisfying $m=m_{i}+n m_{s}$, where $n$ is an integer. This is actually an angular analogue of the much better known phase matching condition for a surface of periodic lattice derived from Floquet's theorem.

The contribution of individual azimuthal channel to optical torque. The timeaveraged optical torque $\langle\boldsymbol{\Gamma}\rangle_{t}$ acting on a scatterer is obtained from the surface integration of the time-averaged angular momentum flux tensor $\langle\overrightarrow{\mathbf{K}}\rangle_{t}$ by

$$
\langle\boldsymbol{\Gamma}\rangle_{t}=-\iint_{\sigma} \mathbf{n} \cdot\langle\overleftrightarrow{\mathbf{K}}\rangle_{t} d S=-\iint_{\sigma} \mathbf{n} \cdot\left(\langle\overleftrightarrow{\mathbf{T}}\rangle_{t} \times \mathbf{r}\right) \mathrm{d} S
$$

where $\sigma$ can be any surface that encloses the entire scatterer, and $\mathbf{n}$ denotes the unit outward normal at surface $\sigma$. The time-averaged Maxwell stress tensor $\langle\overleftrightarrow{\mathbf{T}}\rangle_{t}$ is given by

$$
\langle\overleftrightarrow{\mathbf{T}}\rangle_{t}=\frac{1}{2} \operatorname{Re}\left[\varepsilon_{0} \mathbf{E}_{e} \mathbf{E}_{e}^{*}+\mu_{0} \mathbf{H}_{e} \mathbf{H}_{e}^{*}-\frac{1}{2}\left(\varepsilon_{0} \mathbf{E}_{e} \cdot \mathbf{E}_{e}^{*}+\mu_{0} \mathbf{H}_{e} \cdot \mathbf{H}_{e}^{*}\right) \overleftrightarrow{\mathbf{I}}\right],
$$

where $\varepsilon_{0}$ and $\mu_{0}$ are, respectively, the permittivity and permeability in free space, and $\overleftrightarrow{\mathbf{I}}$ is the unit tensor. The electric (magnetic) field $\mathrm{E}_{e}\left(\mathrm{H}_{e}\right)$ is a sum of incident field $\mathrm{E}_{i}\left(\mathrm{H}_{i}\right)$ 
and scattered field $\mathrm{E}_{s}\left(\mathrm{H}_{s}\right)$, namely, $\mathrm{E}_{e}=\mathrm{E}_{i}+\mathrm{E}_{s}$ and $\mathrm{H}_{e}=\mathrm{H}_{i}+\mathrm{H}_{s}$. These can be expanded in terms of vector spherical wave functions (VSWFs) ${ }^{37,38}$ :

$$
\begin{aligned}
& \mathbf{E}_{i}=-\sum_{l=1}^{\infty} \sum_{m=-l}^{l} i E_{m l}\left[p_{m l} \mathbf{N}_{m l}^{(1)}(k, \mathbf{r})+q_{m l} \mathbf{M}_{m l}^{(1)}(k, \mathbf{r})\right], \\
& \mathbf{H}_{i}=-\frac{k}{\omega \mu} \sum_{l=1}^{\infty} \sum_{m=-l}^{l} E_{m l}\left[q_{m l} \mathbf{N}_{m l}^{(1)}(k, \mathbf{r})+p_{m l} \mathbf{M}_{m l}^{(1)}(k, \mathbf{r})\right], \\
& \mathbf{E}_{s}=\sum_{l=1}^{\infty} \sum_{m=-l}^{l} i E_{m l}\left[a_{m l} \mathbf{N}_{m l}^{(3)}(k, \mathbf{r})+b_{m l} \mathbf{M}_{m l}^{(3)}(k, \mathbf{r})\right] \\
& \mathbf{H}_{s}=\frac{k}{\omega \mu} \sum_{l=1}^{\infty} \sum_{m=-l}^{l} E_{m l}\left[b_{m l} \mathbf{N}_{m l}^{(3)}(k, \mathbf{r})+a_{m l} \mathbf{M}_{m l}^{(3)}(k, \mathbf{r})\right]
\end{aligned}
$$

where $E_{m l}=\left|E_{0}\right| i^{l} \gamma_{m l}^{1 / 2}$ and $\gamma_{m l}=\frac{2 l+1}{l(l+1)} \frac{(l-m) !}{(l+m) !}$. The expansion coefficients $p_{m l}$ and $q_{m l}\left(a_{m l}\right.$ and $\left.b_{m l}\right)$ are known as partial wave expansion coefficients for incident (scattered) waves. Here, $p_{m l}$ and $q_{m l}$ can be obtained from an overlap integral between the incident field and VSWFs. For a collection of spheres $a_{m l}$ and $b_{m l}$ can be obtained by solving the scattering problem using the generalized Mie theory for multispheres.

By the orthogonality of the VSWFs, the integration in Eq.(20) can be worked out analytically (for an arbitrary incident field) at a surface far away from the scatterer to give the $z$-component $\Gamma_{z}$ of the time-averaged optical torque,

$$
\Gamma_{z}=\frac{2 \pi \varepsilon\left|E_{0}\right|^{2}}{k^{3}} \sum_{l=1}^{\infty} \sum_{m=-l}^{l} m \operatorname{Re}\left[p_{m l} a_{m l}^{*}+q_{m l} b_{m l}^{*}-\left(\left|a_{m l}\right|^{2}+\left|b_{m l}\right|^{2}\right)\right] .
$$

On the other hand, with the incident and scattered field expanded in terms of VSWFs, the rate $W_{s c a}$ at which incident wave energy is scattered and the rate $W_{e x t}$ at which the energy is intercepted can be written as, see, e.g., Ref. 38

$$
\begin{aligned}
W_{\text {sca }} & =-\iint_{\sigma} \mathbf{n} \cdot\left\langle\mathbf{P}_{\text {sca }}\right\rangle_{t} d S=\sum_{m} W_{s c a}^{(m)}, \\
W_{\text {ext }} & =-\iint_{\sigma} \mathbf{n} \cdot\left\langle\mathbf{P}_{\text {ext }}\right\rangle_{t} d S=\sum_{m} W_{e x t}^{(m)},
\end{aligned}
$$

where

$$
\begin{aligned}
& W_{s c a}^{(m)}=\frac{2 \pi \varepsilon_{0} c\left|E_{0}\right|^{2}}{k^{2}} \sum_{l}\left(\left|a_{m l}\right|^{2}+\left|b_{m l}\right|^{2}\right) \\
& W_{e x t}^{(m)}=\frac{2 \pi \varepsilon_{0} c\left|E_{0}\right|^{2}}{k^{2}} \sum_{l} \operatorname{Re}\left(p_{m l} a_{m l}^{*}+q_{m l} b_{m l}^{*}\right),
\end{aligned}
$$

and the timed-averaged Poynting vectors $\left\langle\mathbf{P}_{\text {sca }}\right\rangle_{t}$ and $\left\langle\mathbf{P}_{\text {ext }}\right\rangle_{t}$ are given by

$$
\left\langle\mathbf{P}_{\text {sca }}\right\rangle_{t}=\frac{1}{2} \operatorname{Re}\left[\mathbf{E}_{s} \times \mathbf{H}_{s}^{*}\right], \quad\left\langle\mathbf{P}_{\text {ext }}\right\rangle_{t}=\frac{1}{2} \operatorname{Re}\left[\mathbf{E}_{i} \times \mathbf{H}_{s}^{*}+\mathbf{E}_{s} \times \mathbf{H}_{i}^{*}\right] .
$$

For an incident wave from a single azimuthal channel $m_{i}$, the partial wave expansion coefficients satisfy $p_{m l}=p_{m l} \delta_{m m_{i}}$ and $q_{m l}=q_{m l} \delta_{m m_{i}}$, where $\delta_{m m^{\prime}}$ is the Kronecker delta. So Eqs. (23) and (24), together with $W_{\text {ext }}=W_{\text {sca }}$ for lossless scatterer, imply that

$$
\begin{gathered}
\Gamma_{z}=\frac{2 \pi \varepsilon_{0}\left|E_{0}\right|^{2}}{k^{3}}\left[\sum_{l, m} m_{i}\left(\left|a_{m l}\right|^{2}+\left|b_{m l}\right|^{2}\right)-\sum_{l, m} m\left(\left|a_{m l}\right|^{2}+\left|b_{m l}\right|^{2}\right)\right] \\
=\frac{2 \pi \varepsilon_{0}\left|E_{0}\right|^{2}}{k^{3}} \sum_{l, m}\left(m_{i}-m\right)\left(\left|a_{m l}\right|^{2}+\left|b_{m l}\right|^{2}\right) \\
=-\frac{m_{s}}{\omega} \sum_{n=-\infty}^{\infty} n \sum_{l=\max \{|m|, 1\}}^{\infty} W_{m l},
\end{gathered}
$$

where $W_{m l}=\frac{2 \pi \varepsilon_{0} c\left|E_{0}\right|^{2}}{k^{2}}\left(\left|a_{m l}\right|^{2}+\left|b_{m l}\right|^{2}\right)>0$ with $W_{s c a}=\sum_{l, m} W_{m l}$, and $m=m_{i}+$ $n m_{s}$ for a scatterer with $m_{s}$-fold rotational symmetry, see, Eq.(10). The first summation in Eq. (25a) corresponds to the extinction torque, which has the same sign as $m_{i}$, leading definitely to a POT, while the second summation in (25a) corresponds to recoil toque that can be positive or negative, since $m\left(=m_{i}+n m_{s}\right)$ and $m_{i}$ may be of the same or opposite signs. Equation (25c) indicates that in order to exert an optical torque on a lossless object, the latter must scatter the incident wave into azimuthal channel $m$ that differs from the incoming one $m_{i}$ (such that $n \neq 0$ ). Based on Eq. (25c), one can separate the optical torque $\Gamma_{z}$ into partial torque $\tau_{m}$ that is due to each individual azimuthal channel $m$ of the scattered field

$$
\Gamma_{z}=\sum_{m} \tau_{m}=\sum_{n} \tau_{m_{i}+n m_{s}}, \quad \tau_{m_{i}+n m_{s}}=-\frac{m_{s} n}{\omega} \sum_{l=\max \left\{\left|m_{i}+n m_{s}\right|, 1\right\}}^{l_{\max }} W_{\left(m_{i}+n m_{s}\right) l},
$$

where $l_{\max }$ is the truncation order for the angular momentum index in the infinite series. Equation(26) implies that all partial torques associated with positive (negative) $n$ contribute to NOT (POT). The partial torques $\tau_{m}$ for some different allowed $m_{s}$ (with $n= \pm 1, \pm 2, \pm 3$ ) are shown in Fig. 4 for the case where a left circularly plane wave (with $m_{i}=1$ ) impinges on scatterers with $m_{s}=3$ and 6. From Fig. 4, it is found that the absolute values of partial torque due to the same $|n|$ are quite close, since they contain roughly the same number of terms according to Eq.(26). In particular, all terms show oscillatory behavior versus $k R$ due to field retardation. It is this retardation-induced oscillatory behavior that gives rise to the competition between partial torque with positive and negative $n$, resulting in NOT in some intervals of values in $k R$.

1. Allen, L., Beijersbergen, M. W., Spreeuw, R. J. C. \& Woerdman, J. P. Orbital angular momentum of light and the transformation of Laguerre-Gaussian laser modes. Phys. Rev. A 45, 8185-8189 (1992).

2. Allen, L., Barnett, S. M. \& Padgett, M. J. Optical Angular Momentum (IOP Publishing, Bristol, 2003).

3. Grier, D. G. A revolution in optical manipulation. Nature 424, 810-816 (2003).

4. Beth, R. A. Mechanical detection and measurement of the angular momentum of light. Phys. Rev. 50, 115-125 (1936).

5. Franke-Arnold, S., Allen, L. \& Padgett, M. Advances in optical angular momentum. Laser Photonics Rev. 2, 299-313 (2008).

6. Friese, M. E. J., Nieminen, T. A., Heckenberg, N. R. \& Rubinsztein-Dunlop, H. Optical alignment and spinning of laser-trapped microscopic particles. Nature 394, 348-350 (1998).

7. Neale, S. L., MacDonald, M. P., Dholakia, K. \& Krauss, T. F. All-optical control of microfluidic components using form birefringence. Nature Mater. 4, 530-533 (2005).

8. He, H., Friese, M. E. J., Heckenberg, N. R. \& Rubinsztein-Dunlop, H. Direct observation of transfer of angular momentum to absorptive particles from a laser beam with a phase singularity. Phys. Rev. Lett. 75, 826-829 (1995).

9. Simpson, N. B., Dholakia, K., Allen, L. \& Padgett, M. J. Mechanical equivalence of spin and orbital angular momentum of light: an optical spanner. Opt. Lett. 22, 52-54 (1997).

10. Friese, M. E. J., Nieminen, T. A., Heckenberg, N. R. \& Rubinsztein-Dunlop, H. Optical torque controlled by elliptical polarization. Opt. Lett. 23, 1-3 (1998).

11. MacDonald, M. P., Paterson, L., Volke-Sepulveda, K., Arlt, J., Sibbett, W. \& Dholakia, K. Creation and manipulation of three-dimensional optically trapped structures. Science 296, 1101-1103 (2002).

12. Liu, M., Zentgraf, T., Liu, Y. M., Bartal, G. \& Zhang, X. Light-driven nanoscale plasmonic motors. Nature Nanotech. 5, 570-573 (2010).

13. Bishop, A. I., Nieminen, T. A., Heckenberg, N. R. \& Rubinsztein-Dunlop, H. Optical application and measurement of torque on microparticles of isotropic nonabsorbing material. Phys. Rev. A 68, 033802 (2003).

14. Dienerowitz, M., Mazilu, M., Reece, P. J., Krauss, T. F. \& Dholakia, K. Optical vortex trap for resonant confinement of metal nanoparticles. Opt. Exp. 16, 4991-4999 (2008).

15. Friese, M. E. J., Rubinsztein-Dunlop, H., Gold, J., Hagberg, P. \& Hanstrop, D. Optically driven micromachine elements. Appl. Phys. Lett. 78, 547-549 (2001).

16. Paterson, L., MacDonald, M. P., Arlt, J., Sibbett, W., Bryant, P. E. \& Dholakia, K. Controlled rotation of optically trapped microscopic particles. Science 292, 912-914 (2001).

17. Ng, J., Lin, Z. F. \& Chan, C. T. Theory of optical trapping by an optical vortex beam. Phys. Rev. Lett. 104, 103601 (2010).

18. O'Neil, A. T., MacVicar, I., Allen, L. \& Padgett, M. J. Intrinsic and extrinsic nature of the orbital angular momentum of a light beam. Phys. Rev. Lett. 88, 053601 (2002).

19. Courtial, J., Dholakia, K., Allen, L. \& Padgett, M. J. Gaussian beams with very high orbital angular momentum. Opt. Commun. 144, 210-213 (1997).

20. Barnett, S. M. \& Allen, L. Orbital angular momentum and nonparaxial light beams. Opt. Commun. 110, 670-678 (1994).

21. Arita, Y., Mazilu, M. \& Dholakia, K. Laser-induced rotation and cooling of a trapped microgyroscope in vacuum. Nature Commun. 4, 2374 (2013).

22. Dholakia, K. \& Reece, P. Optical micromanipulation takes hold. Nanotoday 1, 18-27 (2006).

23. Dholakia, K., Reece, P. \& Gu, M. Optical micromanipulation. Chem. Soc. Rev. 37, $42-55$ (2008).

24. Chen, J., Ng, J., Lin, Z. F. \& Chan, C. T. Optical pulling force. Nature Photon. 5, 531-534 (2011)

25. Novitsky, A., Qiu, C. W. \& Wang, H. F. Single gradientless light beam drags particles as tractor beams. Phys. Rev. Lett. 107, 203601 (2011).

26. Sukhov, S. \& Doqariu, A. Negative nonconservative forces: optical "tractor beams" for arbitrary objects. Phys. Rev. Lett. 107, 203602 (2011).

27. Brzobohaty, O., Karasek, V., Siler, M., Chvatal, L., Cizmar, T. \& Zemanek, P. Experimental demonstration of optical transport, sorting and self-arrangement using a 'tractor beam'. Nature Photon. 7, 123-127 (2013).

28. Jackson, J. D. Classical Electrodynamics (Wiley, New York, 1999).

29. Tsang, L., Kong, J. A. \& Ding, K. H. Scattering of Electromagneticwaves: Theories and Applications (Wiley, NewYork, 2000).

30. Bohren, C. F. \& Huffman, D. R. Absorption and Scattering of Light by Small Particles (Wiley, NewYork, 1983). 
31. Nieminen, T. A., Parkin, S. J. W., Heckenberg, N. R. \& Rubinsztein-Dunlop, H. Optical torque and symmetry. Proc. SPIE 5514, 254-256 (2004).

32. Nieminen, T. A., Asavei, T., Loke, V. L. Y., Heckenberg, N. R. \& Dunlop, H. R. Symmetry and the generation and measurement of optical torque. J. Quant. Spectrosc. RA. 110, 1472-1482 (2009).

33. Ching, E. S. C., Leung, P. T. \& Young, K. Optical Processes in Microcavities (World Scientific, Singapore, 1996).

34. Newton, R. G. Scattering Theory of Waves and Particles (Springer, New York, 1982).

35. Tsang, L., Kong, J. A., Ding, K. H. \& Ao, C. O. Scattering of Electromagneticwaves: Numerical Simulations (Wiley, NewYork, 2000).

36. Stratton, J. Electromagnetic Theory (McGraw-Hill, New York, 1941).

37. Ng, J., Lin, Z. F., Chan, C. T. \& Sheng, P. Photonic clusters formed by dielectric microspheres: Numerical simulations. Phys. Rev. B 72, 085130 (2005).

38. Xu, Y. L. Electromagnetic scattering by an aggregate of spheres. Appl. Opt. 34, 4573-4588 (1995).

\section{Acknowledgments}

This work is supported by Hong Kong RGC through HKUST2/CRF/11G and AoE/P-02/12. J.N. is also supported by RGC through HKBU604011 and HKBU603312. Z.L. is supported by NSFC through 11174059. J.C. is supported by Shanxi Science and Technology Department through 2014011008-1 and NSFC through 11404201.

\section{Author contributions}

All authors discuss the work thoroughly and extensively. J.C. performed the simulation, and assisted in physical analysis and analytical calculation. J.N. discovered the phenomenon of NOT and interpreted the physics. K.D. assisted in simulation, physical analysis, and analytical calculations. K.H.F. contributed to the initial conception of NOT. Z.F.L. developed the fundamental methodology and formalism. C.T.C. initiated the project and oversaw its progress.

\section{Additional information}

Supplementary information accompanies this paper at http://www.nature.com/ scientificreports

Competing financial interests: The authors declare no competing financial interests. How to cite this article: Chen, J. et al. Negative Optical Torque. Sci. Rep. 4, 6386; DOI:10.1038/srep06386 (2014).

This work is licensed under a Creative Commons Attribution-NonCommercialNoDerivs 4.0 International License. The images or other third party material in this article are included in the article's Creative Commons license, unless indicated otherwise in the credit line; if the material is not included under the Creative Commons license, users will need to obtain permission from the license holder in order to reproduce the material. To view a copy of this license, visit http:// creativecommons.org/licenses/by-nc-nd/4.0/ 